\title{
CELLULARITY AT THE BOUNDARY OF A MANIFOLD'
}

\section{CHARLES GREATHOUSE}

I. Introduction and definitions. A closed subset $X$ of an $n$-manifold $M^{n}$ will be said to be cellular at the boundary (CAB) of $M^{n}$ if there is a sequence $\left\{B_{i}^{n}\right\}$ of closed $n$-cells in $M^{n}$ satisfying: $B_{i}^{n} \cap\left[\operatorname{Bd}\left(M^{n}\right)\right]=B_{i}^{n-1}$ a closed $(n-1)$-cell, $B_{i+1}^{n-1} \subset \operatorname{Int}\left(B_{i}^{n-1}\right)$, $\left[B_{i+1}^{n} \cap \operatorname{Int}\left(M^{n}\right)\right] \subset \operatorname{Int}\left[B_{i}^{n} \cap \operatorname{Int}\left(M^{n}\right)\right]$, and $\bigcap_{i=1}^{\infty} B_{i}^{n}=X$. Thus, the notion of $\mathrm{CAB}$ is the analogue, for subsets intersecting the boundary of a manifold, of the concept of cellularity introduced by Brown in [1].

Theorem II.2 shows that $\mathrm{CAB}$ sets behave like points on the boundary of a manifold. With the aid of a theorem of McMillan's [2], we give criteria for a compact absolute retract to be CAB of a piecewise-linear $n$-manifold for $n \neq 4$. A product theorem for CAB sets is given and with some restrictions on dimensions, we show that subarcs of a $C A B$ arc are either $\mathrm{CAB}$ or cellular subsets of the interior of the manifold.

We assume a familiarity with [2], [3], and [4]. $R^{n}, S^{n}$ denote $n$ space and the $n$-sphere. $D^{n}(j)$ is the closed $n$-ball in $R^{n}$ with center at the origin and radius $j . I^{n}(j)=D^{n-1}(j) \times[0, j]$. The empty set is denoted by $\varnothing$.

Let $A, B$ be subsets of an $n$-manifold $M^{n}$ and let $\delta$ be a positive number. Then $\operatorname{Int}\left(M^{n}\right), \operatorname{Bd}\left(M^{n}\right)$ denote the interior and boundary of $M^{n}$ respectively, $d(A, B)$, the distance from $A$ to $B, \mathrm{Cl}(A)$, the closure of $A$ in $M^{n}$, and $V_{\delta}(A)$, the subset of $M^{n}$ consisting of points $x$ such that $d(x, A)<\delta$.

Let $M^{n}$ be an $n$-manifold with nonempty boundary and let $X$ be a subset of $M^{n}$ such that $X \cap \operatorname{Bd}\left(M^{n}\right) \neq \varnothing$. Then $2 M^{n}$ denotes an $n$ manifold with empty boundary obtained by taking two copies $M_{1}^{n}, M_{2}^{n}$ of $M^{n}$ and identifying corresponding boundary points. Similarly, if $X_{1}, X_{2}$ are the copies of $X$ in $M_{1}^{n}, M_{2}^{n}$ respectively, then $2 X$ is the subset of $2 M^{n}$ consisting of $X_{1} \cup X_{2}$.

\section{The pointlike character of $\mathrm{CAB}$ sets.}

LEMMA II.1. If $X$ is CAB of an $n$-manifold $M^{n}$, a sequence $\left\{\left(B_{t}^{n}\right)^{\prime}\right\}$ of closed n-cells may be picked which satisfy (in addition to the necessary

Presented to the Society, November 21, 1964 under the title $A$ criterion for cellularity at the boundary (CAB) of a manifold; received by the editor November 5, 1964 .

1 Research supported by grant NSF-GP 211. 
conditions for $X$ to be $\mathrm{CAB}$ of $\left.M^{n}\right)$ the following: $\left(B_{i}^{n-1}\right)^{\prime}$ is a flat closed $(n-1)$-cell in $\operatorname{Bd}\left[\left(B_{i}^{n}\right)^{\prime}\right], \quad\left[\left(B_{i}^{n}\right)^{\prime} \cap \operatorname{Int}\left(M^{n}\right)\right] \approx R^{n-1} \times[0,1)$, and $\operatorname{Bd}\left[\left(B_{i}^{n}\right)^{\prime}\right]-\operatorname{Int}\left[\left(B_{i}^{n-1}\right)^{\prime}\right]$ is bicollared in $M^{n}$.

Proof. Let $\left\{B_{i}^{n}\right\}$ satisfy the necessary conditions for $X$ to be CAB of $M^{n}$. We can pick an $(n-1)$-cell $F_{i}^{n-1}$ satisfying: $B_{i+1}^{n-1} \subset \operatorname{Int}\left(F_{i}^{n-1}\right)$ $C F_{i}^{n-1} \subset \operatorname{Int}\left(B_{i}^{n-1}\right)$ and $\operatorname{Bd}\left(F_{i}^{n-1}\right)$ is bicollared in $B_{i}^{n-1}$. Then there is a homeomorphism $h_{i}$ of $B_{i}^{n}$ onto $I^{n}(1)$ such that $h_{i}\left(F_{i}^{n-1}\right)=D^{n-1}(1)$. There is an $\epsilon_{i}, 0<\epsilon_{i}<\frac{1}{2}$, such that $d\left[h_{i}\left(B_{i+1}^{n-1} \cup X\right), \operatorname{Bd}\left[I^{n}(1)\right]\right.$ $\left.-\operatorname{Int}\left[D^{n-1}(1)\right]\right]>\epsilon_{i}$. Take $\left(B_{i}^{n}\right)^{\prime}=h_{i}^{-1}\left[I^{n}\left(1-\epsilon_{i}\right)\right]$. Then $\left\{\left(B_{i}^{n}\right)^{\prime}\right\}$ is the required sequence.

TheOREM II.2. Let $X$ be CAB of an $n$-manifold $M^{n}$ and let $C^{n}$ be a closed $n$-cell in $M^{n}$ satisfying $X \subset C^{n},\left[X \cap \operatorname{Bd}\left(M^{n}\right)\right]=\left[X \cap \operatorname{Bd}\left(C^{n}\right)\right]$ $C \operatorname{Int}\left[C^{n} \cap \operatorname{Bd}\left(M^{n}\right)\right]$. Then there is a map $h$ of $M^{n}$ onto itself such that $h \mid \mathrm{Cl}\left(M^{n}-C^{n}\right)=1, h\left(C^{n}\right)=C^{n}, h(X)=p \in \mathrm{Bd}\left(M^{n}\right)$ and $h \mid M^{n}-X$ is a homeomorphism of $M^{n}-X$ onto $M^{n}-\{p\}$. Thus, $M^{n} / X \approx M^{n}$.

Proof. Take a sequence $\left\{B_{i}^{n}\right\}$ assured by Lemma II.1. We may assume that $B_{1}^{n} \subset C^{n}$ and $\left[B_{1}^{n} \cap \mathrm{Bd}\left(C^{n}\right)\right] \subset \operatorname{Int}\left[C^{n} \cap \mathrm{Bd}\left(M^{n}\right)\right]$. In the manner of the proof of Theorem 1 of [1], we inductively pick a sequence $\left\{h_{i}\right\}$ of homeomorphisms of $M^{n}$ onto itself satisfying: $h_{1} \mid \mathrm{Cl}\left(M^{n}-C^{n}\right)=1$, the diameter of $h_{1}\left(B_{1}^{n}\right)$ is less than $1, h_{i+1} \mid M^{n}-B_{i}^{n}$ $=h_{i} \mid M^{n}-B_{i}^{n}$, and the diameter of $h_{i+1}\left(B_{i+1}^{n}\right)$ is less than $1 / i+1$. Then $h=\lim _{i} h_{i}$ is the required map.

Corollary II.3. Let $\left\{X_{i} \mid i=1, \cdots, k\right\}$ be a finite collection of disjoint subsets of an n-manifold $M^{n}$ such that each $X_{i}$ is either cellular in $\operatorname{Int}\left(M^{n}\right)$ or CAB of $M^{n}$. Then $M^{n} \approx X$, where $X$ is the decomposition space obtained by identifying $X_{i}$ to a point $p_{i}, i=1, \cdots, k$.

\section{III. $\mathrm{CAB}$ criteria for an absolute retract.}

LemMa III.1 ([5, p. 33]). If $A$ is a closed subspace of a metrizable space $X$ and if both $A$ and $X$ are absolute retracts, then $A$ is a strong deformation retract of $X$.

LEMMA III.2 (BoRsUK [6]). Every locally contractible compact metrizable space of finite dimension is an absolute neighborhood retract.

TheоReм III.3. Let $X$ and $Y$ be finite dimensional (metric) compact absolute retracts and let $Y$ be a closed subset of $X$. Then $X / Y$ is a compact absolute retract.

Proof. Obviously, $X / Y$ is compact and finite dimensional. By Theorem 2.2 [7, p. 123], $X / Y$ is a metric space. By Lemma III.1, $Y$ is a strong deformation retract of $X$. Thus, if $f$ is the quotient map 
of $X$ onto $X / Y$ with $f(Y)=y, X / Y$ is contractible to the point $y$. This implies that $X / Y$ is locally contractible and by Lemma III.2, $X / Y$ is an absolute neighborhood retract. Finally, $X / Y$ is a compact absolute retract since it is a compact contractible absolute neighborhood retract.

Theorem III.3 will be applied to situations where $X$ is a compact absolute retract in a manifold $M$ and $Y=X \cap \operatorname{Bd}(M)$ is a compact absolute retract in $\operatorname{Bd}(M)$. Then $X / Y$ is a compact absolute retract in $M / Y$. If $Y$ is $\mathrm{CAB}$ of $M, M / Y \approx M$ and we may assume that $Y$ is a point in $\operatorname{Bd}(M)$ to simplify arguments. In this case, $2 X$ will be a compact absolute retract in $2 M$.

Lemma III.4. Let $M^{n-1}$ be an (n-1)-manifold topologically embedded in the interior of an n-manifold $M^{n}, n>3$. Let $B^{n-1}$ be a closed $(n-1)$ cell in $M^{n-1}, p \in \operatorname{Int}\left(B^{n-1}\right)$, and let $B^{n-1}$ be locally flat in $M^{n}$ except at $p$. Then $B^{n-1}$ is also locally flat at $p$ provided it has a one-sided local collar at $p$.

Proof. Let $B^{n}$ be a closed $n$-cell in $M^{n}$ such that $p \in \operatorname{Int}\left(B^{n}\right) . B^{n-1}$ has a one-sided local collar at $p$, thus, there is a homeomorphism $h: I^{n}(1) \rightarrow \operatorname{Int}\left(B^{n}\right)$ such that $p=h(0), h\left[D^{n-1}(1)\right] \subset \operatorname{Int}\left(B^{n-1}\right)$, and $h\left[I^{n}(1)-D^{n-1}(1)\right] \cap M^{n-1}=\varnothing$. Let $S^{n-1}\left(\frac{1}{2}\right)=h\left[\operatorname{Bd}\left(I^{n}\left(\frac{1}{2}\right)\right]\right.$. Then $S^{n-1}\left(\frac{1}{2}\right)$ is locally flat in $\operatorname{Int}\left(B^{n}\right) \approx R^{n}$ except at $p$. Hence, by the corollary in [8], $S^{n-1}\left(\frac{1}{2}\right)$ is flat in $\operatorname{Int}\left(B^{n}\right)$. This implies that $B^{n-1}$ is locally flat at $p$.

LEMMA III.5. Let $X$ be a compact subset of an n-manifold $M^{n}, n>3$. Then $X$ is $\mathrm{CAB}$ of $M^{n} \Leftrightarrow X \cap \mathrm{Bd}\left(M^{n}\right)=Y$ is a cellular subset of $\mathrm{Bd}\left(M^{n}\right)$ and $X$ is cellular in $2 M^{n}$.

Proof. The necessity follows from the definition of $C A B$ and the fact that $\operatorname{Bd}\left(M^{n}\right)$ is bicollared in $2 M^{n}$. Thus, suppose $Y$ is a cellular subset of $\operatorname{Bd}\left(M^{n}\right)$ and $X$ is cellular in $2 M^{n}$. We consider $X=X_{1}$ a subset of $M_{1}^{n}$, where $2 M^{n}=M_{1}^{n} \cup M_{2}^{n}$ joined along their boundaries. Let $f$ be the quotient map of $2 M^{n}$ onto $2 M^{n} / X$ and let $f(X)=p$. Cellular subsets of the boundary of a manifold are trivially CAB of the manifold since the boundary is collared in the manifold. Therefore, $Y$ is CAB of $M_{2}^{n}$ and by Theorem II.2, $f\left(M_{2}^{n}\right) \approx M_{2}^{n}$. Thus, $f\left[\operatorname{Bd}\left(M_{i}^{n}\right)\right]$ is collared in $f\left(M_{2}^{n}\right)$ and by Lemma III.4, $f\left[\operatorname{Bd}\left(M_{i}^{n}\right)\right]$ is locally flat in $f\left(2 M^{n}\right)$ at $f(X)=p$. Hence, we pick a sequence $\left\{B_{i}^{n}\right\}$ of closed $n$-cells in $f\left(M_{1}^{n}\right)$ satisfying the conditions necessary for $p$ to be CAB of $f\left(M_{1}^{n}\right)$ and such that $f^{-1}\left(B_{1}^{n}\right)$ lies in the interior of some closed $n$-cell in $2 M^{n}$ containing $X$. Then $\left\{f^{-1}\left(B_{i}^{n}\right)\right\}$ is a sequence of closed $n$-cells in $M_{1}^{n}$ satisfying the conditions necessary for $X$ to be CAB of $M_{1}^{n}$. 
TheOREM III.6. Let $X$ be a compact subset of a piecewise-linear $n$ manifold $M^{n}, n>5$, such that $X$ and $X \cap \operatorname{Bd}\left(M^{n}\right)=Y$ are absolute retracts. Then $X$ is CAB of $M^{n} \Leftrightarrow$ for each open set $U$ of $M^{n}$ containing $X$, there is an open set $V$ of $M^{n}$ such that $X \subset V \subset U$ and: (1) each loop in $V-X$ is homotopic in $U-X$ to a loop in $\operatorname{Bd}\left(M^{n}\right)$ and (2) each loop in $(V-X) \cap \operatorname{Bd}\left(M^{n}\right)$ is nullhomotopic in $(U-X) \cap \operatorname{Bd}\left(M^{n}\right)$.

Proof. The necessity is obvious in view of Lemma II.1. Thus, we show the sufficiency. We will do this by showing that $Y$ is cellular in $\operatorname{Bd}\left(M^{n}\right), X$ is cellular in $2 M^{n}$, and applying Lemma III.5. We consider $X=X_{1}$ a subset of $M_{1}^{n}$, where $2 M^{n}=M_{1}^{n} \cup M_{2}^{n}$ joined along their boundaries. Condition (2) together with Theorem 1 of [2] imply that $Y$ is cellular in $\mathrm{Bd}\left(M_{i}^{n}\right)$ and hence simultaneously $\mathrm{CAB}$ of $M_{1}^{n}$ and $M_{2}^{n}$. Theorem III.3 shows that $X / Y$ is a compact absolute retract. Thus, we may assume that $Y=y$ is a point in $\operatorname{Bd}\left(M_{i}^{n}\right)$.

Let $U$ be an open set in $2 M^{n}$ containing $X$. We may assume that $U \cap \mathrm{Bd}\left(M_{i}^{n}\right)$ is an open $(n-1)$-cell since $Y=y$ is a point. Let $U_{1}$ $=U \cap M_{1}^{n}$. Then $U_{1}$ is an open set in $M_{1}^{n}$ containing $X$. By hypothesis, there is a set $V_{1}$ open in $M_{1}^{n}$ such that $X \subset V_{1} \subset U_{1}$ and each loop in $V_{1}-X$ is homotopic in $U_{1}-X$ to a loop in $\operatorname{Bd}\left(M_{i}^{n}\right)$. We may also assume that $V_{1} \cap \operatorname{Bd}\left(M_{i}^{n}\right)$ is an open $(n-1)$-cell whose closure $B^{n-1}$ is a closed $(n-1)$-cell contained in $U_{1} \cap \mathrm{Bd}\left(M_{i}^{n}\right)$. There is a positive number $\epsilon$ and a homeomorphism $h: B^{n-1} \times[0, \epsilon) \rightarrow U \cap M_{2}^{n}$ such that $h \mid B^{n-1} \times 0$ is the inclusion map and $h\left[B^{n-1} \times(0, \epsilon)\right] \subset \operatorname{Int}\left(M_{2}^{n}\right)$. Let $V=V_{1} \cup h\left[\operatorname{Int}\left(B^{n-1}\right) \times[0, \epsilon)\right]$. We will show that any loop in $V-X$ is nullhomotopic in $U-X$.

Let $f: S^{1} \rightarrow V-X$. We assume that $f$ is simplicial and $f\left(S^{1}\right)$ is in general position with respect to $\operatorname{Bd}\left(M_{i}^{n}\right)$. If $f\left(S^{1}\right) \cap M_{1}^{n}=\varnothing$, the result follows trivially. Thus, suppose $f\left(S^{1}\right) \cap M_{1}^{n} \neq \varnothing$. Then $f\left(S^{1}\right) \cap M_{1}^{n}$ consists of a finite number of paths in $V_{1}-X$ with endpoints in $\operatorname{Bd}\left(M_{i}^{n}\right)$. Let $\alpha_{i}$ be one such path with endpoints $p_{i}, \phi_{i}$. Then $p_{i}, \phi_{i}$ can be joined by an $\operatorname{arc} \beta_{i}$ in $\left(V_{1}-X\right) \cap \operatorname{Bd}\left(M_{i}^{n}\right)$. If $l_{i}=\alpha_{i} \cup \beta_{i}$, by hypothesis, $l_{i}$ is homotopic in $U_{1}-X$ to a loop in $\operatorname{Bd}\left(M_{i}^{n}\right)$ and hence is nullhomotopic in $U_{1}-X$ since $U_{1} \cap \operatorname{Bd}\left(M_{i}^{n}\right)$ is an open $(n-1)$-cell. This implies that $\alpha_{i}$ is homotopic in $U_{1}-X$ to $\beta_{i}$ with $p_{i}, \phi_{i}$ fixed throughout the homotopy. Since $V \cap M_{2}^{n}=h\left[\operatorname{Int}\left(B^{n-1}\right) \times[0, \epsilon)\right], f\left(S^{1}\right) \cap M_{2}^{n}$ is homotopic in $V \cap M_{2}^{n}$ to a subset of $\operatorname{Int}\left(B^{n-1}\right)-y$ with the homotopy fixed throughout on $\operatorname{Int}\left(B^{n-1}\right)$. Thus, $f\left(S^{1}\right)$ is homotopic in $U-X$ to a loop in $(U-X) \cap \operatorname{Bd}\left(M_{i}^{n}\right)$ and hence is nullhomotopic in $U-X$. Theorem 1 of [2] implies that $X$ is cellular in $2 M^{n}$ and Lemma III.5 shows that $X$ is $\mathrm{CAB}$ of $M^{n}$.

REMARK. Theorem III. 6 holds for $n=5$ if we replace condition (2) by condition $\left(2^{\prime}\right)$ requiring $Y$ to be a cellular subset of $\operatorname{Bd}\left(M^{n}\right)$. 
Lemma III.7. Let $X$ be a closed subset of $I^{n}(1)$. Then $X$ is CAB of $I^{n}(1) \Leftrightarrow X \cap \mathrm{Bd}\left[I^{n}(1)\right]=Y$ is a cellular subset of $\mathrm{Bd}\left[I^{n}(1)\right]$ and $2 X$ is cellular in $2 I^{n}(1) \approx S^{n}$.

Proof. The necessity is obvious. Thus, we show the sufficiency. As usual, $2 I^{n}(1)=I_{1}^{n}(1) \cup I_{2}^{n}(1)$ joined along their boundaries. We may assume that $Y=y$ is a point of $\mathrm{Bd}\left[I_{i}^{n}(1)\right]$ since $Y$ is CAB of $I_{i}^{n}(1)$. Let $f: 2 I^{n}(1) \rightarrow 2 I^{n}(1) / 2 X \approx S^{n}$ be the quotient map with $f(2 X)=f(y)=p$. Now $f\left[\operatorname{Bd}\left(I_{i}^{n}(1)\right)\right]$ is locally flat in $f\left[2 I^{n}(1)\right]$ except possibly at $p$. If $n \neq 3, f\left[\operatorname{Bd}\left(I_{i}^{n}(1)\right)\right]$ is flat. If $n=3$, either $f\left[I_{1}^{n}(1)\right]$ or $f\left[I_{2}^{n}(1)\right]$ is a closed 3-cell [9]. In either case, we may assume without loss of generality that $f\left[I_{1}^{n}(1)\right]$ is a closed $n$-cell. The completion of the proof follows as in the proof of Lemma III.5.

ThEOREM III.8. Let $X$ be a compact subset of a piecewise-linear 3manifold $M^{3}$ such that $X$ and $X \cap \mathrm{Bd}\left(M^{3}\right)=Y$ are absolute retracts and such that for some open set 0 of $M^{3}$ containing $X$, the pair (0, $\left.0 \cap \mathrm{Bd}\left(M^{3}\right)\right)$ is embeddable in $\left(I^{3}(1), \mathrm{Bd}\left[I^{3}(1)\right]\right)$. Then $X$ is CAB of $M^{3} \Leftrightarrow$ for each open set $U$ of $M^{3}$ containing $X$, there is an open set $V$ of $M^{3}$ with $X \subset V \subset U$ and each loop in $V-X$ is nullhomotopic in $U-X$.

Proof. The hypothesis on 0 allows us to assume that $M^{3}=I^{8}(1)$. $Y$ is cellular in $\mathrm{Bd}\left[I^{3}(1)\right]$ since it is a compact absolute retract in the interior of a 2 -manifold. Hence, we assume that $Y=y$ is a point of $\operatorname{Bd}\left[I^{3}(1)\right]$.

Let $U$ be an open set of $2 I^{3}(1)=I_{1}^{3}(1) \cup I_{2}^{3}(1) \approx S^{3}$ containing $2 X$ $=X_{1} \cup X_{2}$. We may assume that $U$ is symmetric with respect to $I_{1}^{3}(1)$ and $I_{2}^{3}(1)$, and that $U \cap \operatorname{Bd}\left[I_{i}^{3}(1)\right]$ is an open 2-cell. Then by hypothesis and a little care, we obtain an open set $V$ of $2 I^{3}(1)$ such that $V$ is symmetric with respect to $I_{1}^{3}(1)$ and $I_{2}^{3}(1), V \cap \mathrm{Bd}\left[I_{i}^{3}(1)\right]$ is an open 2-cell, $X_{i} \subset V_{i}=\left[V \cap I_{i}^{3}(1)\right] \subset U_{i}=\left[U \cap I_{i}^{3}(1)\right]$, and each loop in $V_{i}-X_{i}$ is nullhomotopic in $U_{i}-X_{i}$.

Let $f: S^{1} \rightarrow V-2 X$. We suppose that $f$ is simplicial and that $f\left(S^{1}\right)$ is in general position with respect to $\operatorname{Bd}\left[I_{i}^{3}(1)\right]$. Then $f\left(S^{1}\right) \cap I_{i}^{3}(1)$ is a finite collection of paths in $V_{i}-X$ with endpoints in $\operatorname{Bd}\left[I_{i}^{3}(1)\right]$. As in the proof of Theorem III.6 we join these endpoints with arcs in $\left(V_{i}-X\right) \cap \operatorname{Bd}\left[I_{i}^{3}(1)\right]$ and obtain a homotopy pulling $f\left(S^{1}\right)$ into $(U-X) \cap \operatorname{Bd}\left[I_{i}^{3}(1)\right] . \quad$ Since $(U-X) \cap \operatorname{Bd}\left[I_{i}^{3}(1)\right] \quad$ and $(V-X) \cap \operatorname{Bd}\left[I_{i}^{3}(1)\right]$ are open 2-cells, there is another homotopy pulling $f\left(S^{1}\right)$ into $\left(V_{1}-X\right) \cap \operatorname{Bd}\left[I_{i}^{3}(1)\right]=(V-X) \cap \operatorname{Bd}\left[I_{i}^{3}(1)\right]$ and then, by hypothesis, it is nullhomotopic in $U_{1}-X$. Thus, by Theorem $1^{\prime}$ of [2], $2 X$ is cellular in $2 I^{3}(1)$, and by Lemma III.7, $X$ is CAB of $I^{3}(1)=M^{3}(1)$. 
Lemma III.9. Let $X$ be a compact subset of a piecewise-linear 3manifold $M^{3}$. Then $X$ is $\mathrm{CAB}$ of $M^{3} \Leftrightarrow X \cap \mathrm{Bd}\left(M^{3}\right)=Y$ is cellular in $\operatorname{Bd}\left(M^{3}\right)$ and $2 X$ is cellular in $2 M^{3}$.

Proof. As usual, the necessity is obvious. Thus, we show the sufficiency. Let $U$ be an open set of $M^{3}$ containing $X$. Then $2 U=U_{1} \cup U_{2}$ is an open set of $2 M^{3}=M_{1}^{3} \cup M_{2}^{3}$ containing $2 X=X_{1} \cup X_{2}$. By hypothesis, there is a closed 3-cell $B^{3}$ such that $2 X \subset \operatorname{Int}\left(B^{3}\right) \subset B^{3} \subset U$. By Theorem 3 of [2], we may assume that $B^{3}$ is a piecewise-linear 3-cell. We also suppose that $\mathrm{Bd}\left(B^{3}\right)$ is in general position with respect to $\operatorname{Bd}\left(M_{i}^{3}\right)$. Then $\operatorname{Bd}\left(B^{3}\right) \cap \operatorname{Bd}\left(M_{i}^{3}\right)$ consists of a finite number of simple closed curves. We may assume that $B^{3}$ has been cut down, by removing inessential simple closed curves on $\operatorname{Bd}\left(B^{3}\right)$, to a submanifold $N^{3}$ such that $2 X \subset \operatorname{Int}\left(N^{3}\right), N^{3} \cap M_{i}^{3}=N_{i}$ is a cube with handles, and $N^{3} \cap \operatorname{Bd}\left(M_{\imath}^{3}\right)=D$ is a disk with holes. If $D$ is a disk, we are through. If $D$ is a disk with $n$ holes, we may "cut one of the handles" of either $N_{1}$ or $N_{2}$ to reduce $D$ to a disk with $(n-1)$ holes. By induction, we obtain a closed 3-cell $\left(B^{3}\right)^{\prime}$ either in $U_{1}$ or $U_{2}$ of the required type to show that either $X_{1}$ is $\mathrm{CAB}$ of $M_{1}^{3}$ or $X_{2}$ is $\mathrm{CAB}$ of $M_{2}^{3}$.

Theorem III.10. Let $X$ be a compact 1-dimensional subset of a piecewise-linear 3-manifold $M^{3}$ such that $X$ and $X \cap \mathrm{Bd}\left(M^{3}\right)=Y$ are absolute retracts. Then $X$ is $\mathrm{CAB}$ of $M^{3} \Leftrightarrow$ for each open set $U$ of $M^{3}$ containing $X$, there is an open set $V$ of $M^{3}$ such that $X \subset V \subset U$ and each loop in $V-X$ is nullhomotopic in $U-X$.

Proof. $2 X$ is a compact absolute retract in $2 M^{8}$. D. R. McMillan pointed out to the author that some neighborhood of $2 X$ is embeddable in $R^{3}$ since $2 X$ is 1 -dimensional. A proof similar to that of Theorem III.8 shows that $2 X$ is cellular in $2 M^{3}$. Again, $Y$ is cellular in $\operatorname{Bd}\left(M^{3}\right)$ and thus Lemma III.9 implies that $X$ is CAB of $M^{3}$.

Theorem III.11. Let $X$ be a compact subset of an $i$-manifold $M^{i}$, $i=1,2$, such that $X$ and $X \cap \operatorname{Bd}\left(M^{i}\right)=Y$ are absolute retracts. Then $X$ is $\mathrm{CAB}$ of $M^{i}$ and hence $M^{i} / X \approx M^{i}$.

Proof. The case $i=1$ is trivial. If $i=2, Y$ is cellular in $\operatorname{Bd}\left(M^{2}\right)$, $2 X$ is cellular in $2 M^{2}$ and an easy argument completes the proof.

IV. CAB sets in products.

LEMma IV.1. Let $N^{n}, M^{m}$ be $n, m$ manifolds respectively such that $\operatorname{Bd}\left(N^{n}\right) \neq \varnothing$ and $\operatorname{Bd}\left(M^{m}\right)=\varnothing$. Then $2\left(N^{n} \times M^{m}\right) \approx\left(2 N^{n}\right) \times M^{m}$.

Proof. $2\left(N^{n} \times M^{m}\right)$ consists of two copies of $N^{n} \times M^{m}$.joined along 
$\operatorname{Bd}\left(N^{n} \times M^{m}\right)=\left[B\left(N^{n}\right) \times M^{m}\right] \cup\left[N^{n} \times \operatorname{Bd}\left(M^{m}\right)\right]$, while $\left(2 N^{n}\right) \times M^{m}$ consists of two copies of $N^{n} \times M^{m}$ joined along $\operatorname{Bd}\left(N^{n}\right) \times M^{m}$.

Theorem IV.2. Let $N^{n}, M^{m}$ be piecewise-linear $n, m$ manifolds respectively such that $\operatorname{Bd}\left(N^{n}\right) \neq \varnothing, \operatorname{Bd}\left(M^{m}\right)=\varnothing$, and $n \geqq 2, m \geqq 1$. Let $X$ be a compact subset of $N^{n}, Z$ a compact subset of $M^{m}$, and suppose $X$, $\left[X \cap \operatorname{Bd}\left(N^{n}\right)\right]=Y$, and $Z$ are absolute retracts. If $m+n \geqq 6$, then $X \times Z$ is $\mathrm{CAB}$ of $N^{n} \times M^{m}$.

Proof. Theorem 8 of [2] implies that $Y \times Z$ is cellular in $\operatorname{Bd}\left(N^{n}\right)$ $\times M^{m}=\operatorname{Bd}\left(N^{n} \times M^{m}\right)$. It also implies that $X \times Z$ is cellular in $\left(2 N^{n}\right)$ $\times M^{m}$. Then Lemma IV.1 implies that $X \times Z$ is cellular in $2\left(N^{n} \times M^{m}\right)$. Hence by Lemma III.5, $X \times Z$ is CAB of $N^{n} \times M^{m}$.

A couple of applications of the corollary to Theorem 8 in [2] together with Lemma III.7 give the following theorem.

TheOREM IV.3. Let $X$ be a compact subset of $D^{n}(1)$, such that $X$ and $X \cap \mathrm{Bd}\left[D^{n}(1)\right]$ are absolute retracts. Then $X=X \times 0$ is $\mathrm{CAB}$ of $D^{n}(1) \times[-1,1]$.

V. CAB arcs. Let $\alpha$ be the arc described in Example 1.3 of [10]. We suppose that $\alpha \subset I^{3}(1)$ and $\alpha \cap \operatorname{Bd}\left[I^{3}(1)\right]=\{p\}$, where $p$ is the "good" endpoint of $\alpha$. Then $\alpha$ is the monotone union of subarcs each of which is cellular in Int $\left[I^{3}(1)\right]$ and each of which contains the "bad" endpoint of $\alpha$, but $\alpha$ is not CAB of $I^{3}(1)$ since $2 \alpha$ is not cellular in $2 I^{3}(1)$.

However, going in the other direction we have the following theorem.

THEOREM V.1. Let $\alpha$ be an arc CAB of an $n$-manifold $M^{n}$ and let $\beta$ be a subarc of $\alpha$. Then the following hold:

(1) $\beta \subset \mathrm{Bd}\left(M^{n}\right), n \neq 5 \Rightarrow \beta$ is cellular in $\mathrm{Bd}\left(M^{n}\right)$ and hence $\mathrm{CAB}$ of $M^{n}$,

(2) $\beta \cap \mathrm{Bd}\left(M^{n}\right)$ is a point $(\varnothing), n \neq 4 \Rightarrow \beta$ is $\mathrm{CAB}$ of $M^{n}$ (cellular in $\left.\operatorname{Int}\left(M^{n}\right)\right)$,

(3) $\beta \cap \operatorname{Bd}\left(M^{n}\right)$ is a proper subarc of $\beta, n \neq 4,5 \Rightarrow \beta$ is $\mathrm{CAB}$ of $M^{n}$.

Proof. Since $\alpha$ is CAB of $M^{n}$, we may assume that $M^{n}=I^{n}(1)$. By Lemma III.7, $\alpha \cap \operatorname{Bd}\left[I^{n}(1)\right]=\sigma$ is cellular in $\operatorname{Bd}\left[I^{n}(1)\right] \approx S^{n-1}$ and $2 \alpha$ is cellular in $2 I^{n}(1) \approx S^{n}$. Also $\sigma$ is CAB of $I^{n}(1), \sigma$ is cellular in $2 I^{n}(1)$, and hence $2(\alpha / \sigma)$ is cellular in $2\left(I^{n}(1) / \sigma\right) \approx 2 I^{n}(1) \approx S^{n}$. Theorem 6 of [2] together with Lemma III.7 give (1) and (2) immediately and (3) follows with an additional easy argument. 


\section{REFERENCES}

1. M. Brown, $A$ proof of the generalised Schoenfiies theorem, Bull. Amer. Math. Soc. 66 (1960), 74-76.

2. D. R. McMillan, Jr., A criterion for cellularity in a manifold, Ann. of Math. 79 (1964), 327-337.

3. J. R. Stallings, The piecewise-linear structure of euclidean space, Proc. Cambridge Philos. Soc. 58 (1962), 481-488.

4. M. Brown, Locally flat imbeddings of topological manifolds, Ann. of Math. 75 (1962), 331-341.

5. S. T. Hu, Homotopy theory, Academic Press, New York, 1959.

6. K. Borsuk, Über eine Klasse von lokal susammenhangenden Rdumen, Fund. Math. 19 (1932), 220-240.

7. G. T. Whyburn, Analytic topology, Amer. Math. Soc. Colloq. Publ. Vol. 28, Amer. Math. Soc., Providence, R. I., 1942.

8. J. C. Cantrell, Almost locally flat embeddings of $S^{n-1}$ in $S^{n}$, Bull. Amer. Math. Soc. 69 (1963), 716-718.

9. O. G. Harrold, Jr. and E. E. Moise, Almost locally polyhedral spheres, Ann. of Math. (2) 57 (1953), 575-578.

10. E. Artin and R. H. Fox, Some wild cells and spheres in three-dimensional space, Ann. of Math. 49 (1948), 979-990.

The University OF TenNessee aNd

VANDERBILT UNIVERSITY 